\title{
6.2. FLUCTUATIONS IN THE MOTION OF THE MEAN POLE AND THE ROTATION OF THE EARTH
}

\author{
H. J. ABRAHAM \\ (Mount Stromlo and Siding Spring Observatory, \\ Australian National University)
}

\section{ABSTRACT}

Explanations of the progressive and librational motions of the pole are attempted.

$$
\text { RÉSUMÉ }
$$

On essaye une explication du mouvement progressif et périodique du pôle.

The observed secular motion of the mean pole shows a progressive component and periodic librations, either real or apparent. Evidence is given that real librations should occur.

The total deformation excitation $\Psi$ due to yielding of the Earth should be proportional to $k d$, where $k$ is the Love number and $d$ is the distance of the pole of rotation from the pole of figure. Furthermore, the change in $\tau$, the free nutation period, if due only to yielding of the Earth, should be a simple function of $k$. However, $\tau$ varies with $d$ and therefore $\Psi$ may include a function of $d^{2}$. The value of $d$ is given by the free nutation term $m_{0} \exp \left(i \sigma_{0} t\right)$ plus the forced nutation terms $m \exp (i \sigma t)$ and $n \exp (-i \sigma t)$. Then it can be shown that these lead to mean excitations which vary as $\cos \left(\sigma_{0} \pm \sigma\right) t / 2$, and that one of these produces an excitation that varies as $\cos \left(\sigma-\sigma_{0}\right) t / 4$. The frequencies $\left(\sigma-\sigma_{0}\right) / 2$ and $\left(\sigma-\sigma_{0}\right) / 4$ correspond to the observed libration periods of about 12 and 24 years.

The calculated meridian of the librations is in satisfactory agreement with the observed meridian, $\lambda_{\mathrm{L}}$. The angle between $\lambda_{\mathrm{L}}$ and $\lambda_{\mathrm{p}}$, the meridian of the progressive motion, is given by $\lambda=\cot ^{-1}\left(k / k_{f} \cot \theta\right)$, where $k_{f}$ is the value of $k$ for hydrostatic equilibrium, and $\theta$ is the angle between $\lambda_{p}$ and the major axis of the seasonal excitation.

The amplitude of the librations, and apparent fluctuations in the progressive motion, both vary with $\sigma_{0}$ in a manner to be expected. When the natural frequency $\sigma_{0}$ approaches the forced frequency $\sigma$ the excitations are enhanced, amplitudes become greater, and the mean pole is advanced in the direction of the progressive motion.

Changes in $T$, the length of the day, show an effective value of $k$ apparently varying with $d$. The observed value of $T$ and the amplitude of the polar motion since 1955 show 
similar '6-yearly' variations proportional to $\left|\cos \left(\sigma-\sigma_{0}\right) t / 2\right|$. The range of these variations in $T$ relative to atomic time was $3 \times 10^{-9}$.

The effective value of $k$ (and hence of $\tau$ ) depends inversely on the mean excitation. When the librational excitation grows larger the values of $\tau$ grow smaller than otherwise. Moreover $\lambda$ then grows larger, as it should because effectively $k$ grows smaller. The '6-yearly' excitation which is caused by the resultant of the free and forced nutations shows a similar effect; when the resultant is greatest the excitations which it causes are greatest and the values of $\tau$ are least.

These relationships point to periodic excitations that connect the nutations with the librations and with changes in $T$. 